6 Grußwort

8 Fahr Rad! Die Rückeroberung der Stadt Eine Einführung

16200 Jahre Positionskampf:

Das Fahrrad im Stadtverkehr

- Thomas Kosche

22 Grüne Infrastruktur.

Eine Positionsbestimmung

- Till Rehwaldt

\section{NEW YORK}

28 Mit der Veränderung der Straße

die Welt verändern

$$
\text { - Janette Sadik-Khan }
$$

34 Manhattan Waterfront Greenway

38 Battery Bikeway

40 Big U

42 Randall's Island Hell Gate

Pathway und Connector

46 Masterplan des South Bronx Greenway

48 Columbia Street Bikeway

50

Erfahrungen eines Stadtplaners

- Kees Christiaanse

\section{GRONINGEN}

56

In Groningen spielt das Auto eine Nebenrolle

- Renate van der Zee und

Marco te Brömmelstroet

60

Stadsbalkon
Wem gehört der öffentliche Raum?

- Barbara Lenz

\section{BARCELONA}

68 Barcelona gegen den Klimawandel

- Mercedes Vidal Lago

$72 \quad$ Umgestaltung des Passeig

de St Joan

76 Garcia Fària Promenade

78 Superblocks

80 Gran Via de les Corts Catalanes

$82 \quad$ In der Stadt unterwegs

- Steffen de Rudder

\section{RUHRGEBIET}

88 Essen: Der Weg zur Fahrradstadt

$$
\text { - Simone Raskob }
$$

92 Radschnellweg Ruhr RS1

96 Fuß- und Radwegbrücke Hafen

Grimberg

98 Das Fahrrad als Schlüssel zur urbanen Verkehrswende

- Ludger Koopmann

\section{OSLO}

104 Oslo wird zum Radparadies

- Laura Bliss

110 Oslo City Bike

112 Akrobaten Brücke

114 Dronning Eufemia's Gate

116 FutureBuilt

118 Radeln in der Stadt

- Christiane Thalgott 


\section{PORTLAND}

124 Von Durchschnitt zu Herausragend

$$
\text { - Leah Treat }
$$

128 Tilikum Crossing

130 Max Orange Line

134 Vera Katz Eastbank Promenade

136 Überlegungen zum Stellenwert urbanen Grüns

- lan Mell und Hendrik Behnisch

\section{KARLSRUHE}

142 Von der Fahrradstadt zur Stadt

der Nachbarschaften

- Markus Neppl

146 Mit dem Fahrrad zur Verkehrswende

- Ulrike Reutter

\section{KOPENHAGEN}

152 Fahrradstadt Kopenhagen -

Ein radpolitischer Vorreiter

- Klaus Bondam

156 Cykelslangen

158 Mærsk Tower

160 Butterfly Brücke

162 Nørreport Station

166 Åbuen Brücke

168 Cirkelbroen

170 Supercykelstier

172 Nachdenken über Städtebau

- Ein Interview mit Jan Gehl

\section{PROJEKTE}

184 Cuyperspassage / Museumstraat

(Amsterdam, Niederlande)

190 Lightpath - Te Ara i Whiti

(Auckland, Neuseeland)

194 Peter-Merian- und Jacob-Burckhardt-Haus

(Basel, Schweiz)

198 Idee einer Radbahn (Berlin, Deutschland)

202 Rafting Brücke (Celje, Slowenien)

204 Linearer Park The 606

(Chicago, USA)

208 Hovenring (Eindhoven, Niederlande)

210 Buffalo Bayou Park (Houston, USA)

216 Fahrradparkhaus, Sykkelhotell

(Lillestrøm, Norwegen)

218 Bürogebäude Alphabeta

(London, Großbritannien)

222 Radschnellwege Cycle Superhighways

(London, Großbritannien)

224 Park Madrid RIO (Madrid, Spanien)

228 Masterplan Raggi Verdi

(Mailand, Italien)

232 Fahrradpavillon (Mainz, Deutschland)

234 Wohnhaus und Hotel Ohboy

(Malmö, Schweden)

238 Jim Stynes Brücke (Melbourne, Australien)

240 Quartier DomagkPark (München, Deutschland)

244 Radweg Lafitte Greenway (New Orleans, USA)

246 Byens Bro Brücke (Odense, Dänemark)

250 Küstenradweg Riviera dei Fiori (Von Ospedaletti nach San Lorenzo al Mare, Italien)

254 Friedrich Bayer Brücke (São Paulo, Brasilien)

256 Fahrradparkhaus am Bahnhof und Moreelse Brücke (Utrecht, Niederlande)

262 Dafne Schippers Brücke (Utrecht, Niederlande)

264 Nordbahntrasse (Wuppertal, Deutschland)

268 Bicycle Skyway (Xiamen, China)

272 Weiterführende Literatur

273 Biografien

277 Bildnachweis

278 Impressum 
